using the Systemic Lupus Erythematosus Activity Questionnaire (SLAQ). Multiple significant correlations of fatigue could be demonstrated with age, gender, sleep disorders, pain, physical activity, disease activity, obesity, NSAIDs intake, physical and psychological well-being, overall health status and restrictions on everyday life. The 5 strongest associations with fatigue are given in the table.

Based on these results, further research should be conducted to analyse the mentioned factors in more detail. It remains an interesting hypothesis that therapy aimed to improve one or more associated factors identified will also improve patients' reported fatigue.

\section{PS8:159 MOVING WITH THE TIMES: SOCIAL MEDIA USE AMONGST LUPUS PATIENTS}

LM Wheeler, A Pakozdi, R Rajakariar, M Lewis, A Cove-Smith, D Pyne. Barts Lupus Centre, Barts Health NHS Trust, London, UK

\subsection{6/lupus-2018-abstract.202}

Background Over the last decade the rise in social media use has been almost exponential. There are numerous online platforms where patients can interact for support and information gathering. A study presented at the ACR in 2013 showed lupus patients were willing to participate in Facebook chats for the purposes of disease education. ${ }^{1}$ We sought to discover current usage of social media amongst an ethnically mixed population attending an inner city tertiary lupus centre.

Methods Questionnaires were distributed to consecutive patients attending the Barts Lupus Centre from October 2016 to February 2017. 17 questions asked about patient demographics and patients use of online information and support services, particularly social media platforms, with regards to lupus.

Results 84 completed questionnaires were returned. 83\% respondents were female. There were 28 South Asians, 26 Whites and 24 Blacks, 2 of other ethnic groups and 2 of mixed race. $64 \%(n=54)$ of patients accessed online lupus information and support sites. $45 \%$ percent $(n=38)$ of patients reported using social media sites (26\% South Asians, 34\% Blacks and $34 \%$ Whites). Of those using social media $22 \%$ $(n=8)$ patients used these tools daily and 30\% $(n=11)$ reported weekly use. Facebook $(n=20)$, blogs $(n=9)$, youtube $(n=9)$, and Instagram $(n=7)$ were cited as the most frequently used applications. Most patients $(n=30)$ sought information on the disease, $17(45 \%)$ wanted to find out about new treatments for lupus, 16 (42\%) sought new ways to self-manage their disease, 14 (37\%) sought interactions with other patients, and $10(26 \%)$ were seeking support online. Patients most commonly wanted information on skin and joint complaints and family planning. 66\% $(n=56)$ thought their rheumatology team should have an online social media application to communicate with their patients.

Conclusions A significant proportion of our lupus patients (45\%) use social media to access information and support for their disease. Facebook, blogs, Instagram and Youtube are commonly used. Social media applications can provide physicians with a tool to interact with lupus patients to improve accessibility to health care and better health outcomes.

\section{REFERENCE}

1. Spring NH. 2013 ACR/ARHP Annual Meeting, abstract 990.

\section{PS8:160 HEALTH CARE RESOURCE USE (HRU) AND MEDICAL COST ANALYSES AS A FUNCTION OF SYSTEMIC LUPUS ERYTHEMATOSUS (SLE) DISEASE SEVERITY: ANALYSIS OF CLAIMS DATA OF A GERMAN SICKNESS FUND}

${ }^{1}$ ER Hammond, ${ }^{2} \mathrm{H}$ Freidel, ${ }^{2} \mathrm{E}$ Garal-Pantaler, ${ }^{3} \mathrm{M}$ Pignot, ${ }^{4} \mathrm{E}$ Velthuis, ${ }^{1} \mathrm{X}$ Wang, ${ }^{5} \mathrm{H}$ Nab, ${ }^{1} \mathrm{~B}$ Desta, ${ }^{6} \mathrm{~A}$ Schwarting. ${ }^{1}$ AstraZeneca, Gaithersburg, MD, USA; ${ }^{2}$ Team Gesundheit GmbH, Essen, Germany; ${ }^{3}$ Kantar Health GmbH, Munich, Germany; ${ }^{4}$ Evidera PPD, Utrecht Area, The Netherlands; ${ }^{5}$ AstraZeneca, Cambridge, UK; ${ }^{6}$ Universitätsmedizin der Johannes GutenbergUniversität Mainz, Germany

\subsection{6/lupus-2018-abstract.203}

Purpose Real-world HRU and medical cost analyses support drug development in SLE. The economic burden of SLE as a function of disease severity was quantified for a German cohort.

Methods HRU and cost analyses were performed for patients ( $\geq 18$ years old) with SLE and compared with those of matched controls for 2009-2014 using the Betriebskrankenkassen (BKK) German Sickness Fund Database. BKK is a branch of the statutory German health insurance. SLE was confirmed in 2009 using the diagnosing physician's specialty, repeated SLE-related claims, co-diagnosis codes, laboratory tests, or prescriptions. At least 3 years' data was also required prior to 2009. SLE cases were control matched by age, sex, and baseline Charlson comorbidity index (CCI). Continuous outcomes were compared with nonparametric tests.

Results 1160 patients (mean age: 52 years; median baseline CCI: 2 [females], 3 [males]; baseline CCI range: 1-13) with SLE met all inclusion criteria. Most patients were female (84\%) and were diagnosed with SLE before 2009 (85\%). In 2009, SLE prevalence was $37.32 / 100,000$ and incidence was 5.96/100,000. Prevalence increased progressively to 47.36 100,000 in 2014. SLE disease severity was characterised as mild, moderate, and severe for 148, 484, and 528 patients, respectively, per ICD-10-GM and medication/procedures codes. Patients with moderate and severe SLE had greater mean annual medical costs in 2009-2014 than matched controls (e. g., in 2009, moderate SLE: $€ 4867$ vs $€ 3,380, \mathrm{p}<0.0001$; severe SLE: $€ 10001$ vs $€ 4,239$, p<0.0001). Mean costs and number of outpatient visits, hospital stays, outpatient prescriptions and other benefits, and total number of hospital days were significantly greater for the full SLE population and moderate and severe SLE subpopulations than for matched controls. For example, mean costs for hospital stays, outpatient prescriptions, and other benefits in 2009 were $€ 4335$ vs $€ 1,414$, $€ 2582$ vs $€ 1,087$, and $€ 1068$ vs $€ 691$, respectively, for patients with severe SLE vs controls.

Conclusions Economic burden was greater for patients with moderate to severe SLE than for matched controls. Patients with SLE had greater HRU and costs than matched controls. HRU increased with increasing SLE disease severity. New therapeutics that decrease disease activity could reduce economic burden.

\section{PS8:161 THE DISEASE BURDEN IN PATIENTS WITH LONGSTANDING SYSTEMIC LUPUS ERYTHEMATOSUS: FOCUS ON HEALTH RESOURCE USE AND COSTS}

${ }^{1} \mathrm{C}$ Tani, ${ }^{2} \mathrm{~V}$ Lorenzoni, ${ }^{1} \mathrm{R}$ Vagelli, ${ }^{1} \mathrm{C}$ Stagnaro, ${ }^{1} \mathrm{E}$ Elefante, ${ }^{1} \mathrm{~L}$ Carli, ${ }^{2} \mathrm{G}$ Turchetti, ${ }^{1} \mathrm{M}$ Mosca. ${ }^{1}$ Rheumatology Unit, University of Pisa, Italy; ${ }^{2}$ Management Institute, Sant'Anna - Scuola Universitaria Superiore, Pisa, Italy

10.1136/lupus-2018-abstract.204 
Introduction As a consequence of increased SLE patients survival, patients with long disease duration represent a significant proportion of our cohorts. This study aims to evaluate health resource use and the 6 months costs in patients with SLE with long disease duration.

Methods The economic evaluation was performed in terms of cost-of-illness analysis as part of a larger study enrolling SLE patients with at least 15 years of disease duration regularly followed at our unit. At enrollment, the following information were collected: disease activity (SLEDAI), organ damage (SLICC-DI score), comorbidities, treatment patterns; in addition to clinical data, patients were required to complete an ad-hoc questionnaire for the collection of facts relevant for the estimation of the economic dimension and covering the previous six-months. Such a time frame was considered to be appropriate as recall period. Direct health (drugs, hospitalizations, emergency visits, specialists visits, laboratory tests and instrumental examination) and non-health costs (transportation and accommodation) as well as indirect costs because of productivity loss were estimated.

Results 51 adult patients with long disease duration were recruited $(98 \%$ female, mean age $49 \pm 11$ years, median disease duration 17 years, IQR 15-23). Median (IQR) SLEDAI score was $2(0-4)$, median SLICC-DI was $1(0-2)$. The median (IQR) direct health costs per patients over the previous 6 months resulted $410 €(201-1687)$; indirect costs because of productivity lost were $130 €(0-356)$. The median overall cost to the Society was $473 €(327-2148)$; the presence of comorbid conditions resulted associated with higher overall cost for the Society (552€ [327-1807] vs 264€ [94-1164] p=0.046); disease activity and damage at enrollment were not associated with costs increase in this cohort.

Conclusions This cohort of patients with long lasting disease is characterised by low disease activity and mild organ damage; in this setting, the disease burden on the single patient and family is significant and the costs to the Society are influenced by the presence of comorbidities.

\begin{tabular}{|c|c|c|c|}
\hline \multicolumn{4}{|c|}{ Abstract PS8:161 Table 1} \\
\hline & 0 & 1 & 2 \\
\hline $\begin{array}{lr}\text { Hospitalizations } & \text { due } \\
\text { to SLE } & \text { or } \\
\text { complications } & \end{array}$ & $40(78.4 \%)$ & $10(19.6 \%)$ & $1(2 \%)$ \\
\hline \multirow[t]{2}{*}{$E R$ visits } & $45(88.2 \%)$ & $6(11.7 \%)$ & 0 \\
\hline & Modian & $\begin{array}{l}\text { Interquartile } \\
\text { range }\end{array}$ & \\
\hline Specialist visit & 1 & 1.3 & \\
\hline Instrumental exams & 2 & $1 \cdot 3$ & \\
\hline Laboratory exams & 2 & 1.4 & \\
\hline
\end{tabular}

\section{PS8:162 DISEASE PATTERN OF MALE LUPUS}

N Klyukvina, E Nasonov. V.A.Nasonova Research Institute of Rheumatology, Moscow, Russia

\subsection{6/lupus-2018-abstract.205}

Introduction Systemic lupus erythematosus (SLE) is a multisystem autoimmune disease that occurs primarily in women $(9: 1$ compared to men). Available data argue that gender and sex hormones modify susceptibility to, and expression of SLE.
Subjects and methods One hundred and fifty male patients (pts) aged 15 to 64 years (mean age 30, 5 y) with a valid diagnosis of SLE who had been followed up for 15 years were assessed. Results: The mean age at onset was 20 year (range 6-62 years). The distribution of disease onset by decade of age was following: between the ages of 6-20 y - 80 pts (53\%), 21-40 y - 49 pts (33\%), 41-64 y - 21 pts (14\%). Mean duration from the onset to time of diagnosis valid SLE was 12 month (range 1-336). 77 pts were diagnosed during first year from disease onset. 35 pts had delay of true diagnosis for 5 and more years. The most common manifestations for men at disease onset were arthritis (50\%), renal disease $(16,7 \%)$ and haematological abnormalities $(23,3 \%)$. During follow-up we observed malar rash (58\%), arthritis $(73,3 \%)$, renal disease $(54,7 \%)$, CNS involvement $(49,4 \%)$, photosensitivity (51,3\%), discoid lupus (14\%), mucosal ulcers (28\%), serositis $(32,7 \%)$, haematological (72\%) and immunological $(93,3 \%)$ abnormalities.

41 male pts $(27,3 \%)$ satisfied classification Sapporo criteria of definite antiphospholipid syndrome (APS)/Vascular thrombosis developed in 41 pts: 26 pts had only venous thrombosis, 8 pts - merely arterial thrombosis, 7 pts $-\mathrm{a}+\mathrm{v}$ thrombosis. Pts with APS had higher frequency of livedo reticularis (13 pts, 31,7\%), pulmonary hypertension (8 pts, 19,5\%) and heart valve disease (20 pts, 48,8\%) in comparison to pts without APS (11,9\%, 6,5\%, 16,6\% respectively).

Conclusion The present investigation do not confirm older age at disease onset among men with SLE: one half of our pts were younger $20 \mathrm{y}$ at disease onset. We found high frequency of arthritis, renal disease and neuropsychiatric manifestations over the course of illness. CNS involvement was the rare initial symptom. Men with SLE often had delay of valid diagnosis of SLE. We observed high incidence of definite APS in men with SLE.

\section{PS8:163 CHARACTERISTICS AND OUTCOME OF LUPUS NEPHRITIS IN MOROCCO}

I Haddiya. Faculty of Medicine, University Mohamed First, Oujda, Morocco

\subsection{6/lupus-2018-abstract.206}

There is wide variation in clinical presentation and outcome of lupus nephritis (LN) among different ethnic groups. Few data for LN exist on North Africans, especially those from Morocco. The aim of our study was to review retrospectively the features and outcome of LN in Moroccan patients.

Patients and methods We performed a multicentric retrospective study. A total of 209 patients with LN were included. All patients met American Rheumatism Association criteria. LN was classified according to the International Society of Nephrology/Renal Pathology Society classification. We adopted previously defined outcome criteria for LN.

Results There were 185 females and 24 males, with a mean age of 27.2 years. At first presentation, we noted hypertension in $31 \%$, hematuria in $72 \%$, nephrotic syndrome in $47 \%$, and renal failure in $64 \%$ of cases. Renal biopsy revealed predominant proliferative classes in more than $80 \%$ of patients. Patients received different regimens mainly based on intravenous cyclophosphamide and Mycophenolate. After a mean follow-up of 22 months, remission occurred in 46.7\%, relapses in $81 \%$, end-stage renal failure in $24 \%$, and death in $13 \%$ of 